\title{
Design of an Adaptive Boost Energy-Saving Fuzzy Control System Driven by the Finite State Machine
}

\author{
Wen Ren (iD, Xia Wen (D, and Sencai Lai $(\mathbb{D}$ \\ Sanming University, College of Mechanical and Electrical Engineering, Sanming, Fujian 365004, China \\ Correspondence should be addressed to Wen Ren; auwren@foxmail.com
}

Received 7 March 2021; Revised 18 April 2021; Accepted 26 April 2021; Published 8 May 2021

Academic Editor: G. Muhiuddin

Copyright (C) 2021 Wen Ren et al. This is an open access article distributed under the Creative Commons Attribution License, which permits unrestricted use, distribution, and reproduction in any medium, provided the original work is properly cited.

\begin{abstract}
Aiming at the challenging problem of the traditional warp knitting machine electronic jacquard control system with complex structure of multiple circuit boards layered cascade, such as large physical space occupation, high power consumption, and independent high-voltage power supply voltage, we proposed an embedded circuit and control strategy design for the piezoelectric jacquard needle (PJN) with adaptive boost and energy recovery functions. Firstly, the electromechanical dynamics model of PJN was established. Secondly, the fuzzy PI double closed-loop control algorithm driven by a finite state machine is proposed. Thirdly, with the help of a Metal-Oxide-Semiconductor Field-Effect Transistor (MOSFET), the PJN is integrated with the drive circuit. The drive circuit of PJN uses an energy storage inductor to replace the current limiting resistor of the traditional drive circuit, which can not only limit the forward charging current of the PJN and reduce energy loss but also can use the energy absorbed from the low-voltage power supply to adaptively boost the power supply of the PJN to the high voltage required for working conditions. The simulation results show that the new PJN drive circuit has an adaptive self-boost function. The PWM signal modulated by the fuzzy PI double closed-loop control algorithm can efficiently and accurately control the adaptive boost power supply and the voltage across the PJN. The mode of the circuit can be correctly switched through the sequential logic of the finite state machine and realize the energy recovery function.
\end{abstract}

\section{Preface}

At present, traditional motors and electromagnetic drives can no longer meet the drive requirements of special equipment for precision motion [1]. A new generation of intelligent actuators represented by piezoelectric ceramic actuators, giant magnetostrictive actuator, and shape memory alloy actuators [2-4] are not only widely used in nanolevel intelligent precision manufacturing equipment in the fields of aerospace, bioengineering, etc. but also gradually used in traditional industrial automation control fields, such as textile equipment [5]. At present, these intelligent actuators generally contain embedded circuit systems inside, which can run intelligent control algorithms such as fuzzy control [6-9] and neural network control [10].The research results on the application of piezoelectric ceramics in highprecision motion and positioning control mainly include the establishment of nonlinear models and their compensation mechanisms based on the inherent nonlinear hysteresis and creep characteristics of piezoelectric ceramics, as well as the design of micromovement flexible mechanisms, etc. [11-13]. The research on the application of piezoelectric ceramics in the field of electronic information mainly includes piezoelectric vibration control and energy harvesting, as well as the design of new piezoelectric sensors and driving power supplies [14-16]. When driving the microdisplacement mechanism, the piezoelectric ceramic driver can be regarded as a capacitor [17]; it consumes less energy, but when driving the load, it will generate a larger current in the drive circuit, and there is large power consumption and the problem of low efficiency. In addition, the traditional piezoelectric control system needs to be additionally equipped with special power supplies of different specifications according to the characteristics of the controlled object, resulting in a large system, low integration, and high complexity and cost. 
"Jacquard" is an important pattern knitting method in warp knitting production. The jacquard control method of warp knitting machine has developed from the early mechanical faucet type and electromagnetic field type to the piezoelectric ceramic type. At present, the piezoelectric ceramic jacquard technology that can accurately and independently control each yarn guide needle in the warp knitting machine is developing rapidly. Piezoelectric jacquard needles (PJNs) realize the three-dimensional, rich and complex jacquard effect of warp knitted fabrics. The PJN is a revolutionary change to the traditional electronic traverse jacquard technology and has received extensive attention from the textile academic and engineering circles. Kumaravelu et al. [18] designed a cardless jacquard system based on the TTL logic circuit. The German Karl Mayer company designed and produced a series of high-speed jacquard warp knitting machines [19]. The research team of the 26th Research Institute of China Electronics Technology Group Corporation conducted a systematic study on the warp knitting machine jacquard modification based on the performance analysis of the PJN and the three-dimensional design method [20]. Cheng and Jiang [21] developed a warp knitted fabric design and simulation software for the warp knitting machine jacquard control system. Gao and Liu [22] researched the communication system, control circuit, and management system of the jacquard warp knitting machine. However, the abovementioned research results are limited to the traditional system control architecture, that is, the communication and control system of the piezoelectric jacquard warp knitting machine is composed of a PLC controller, communication relay board, piezoelectric drive board, jacquard needle block, etc., whose system structure is complex, maintenance is difficult, and reliability is low. For example, for a warp knitting machine (model: RDPJ5/1, width: 138 inches), there are 5 bars in total, and 1 to 2 bars with jacquard function are generally configured. The number of jacquard needles configured in one bar is up to 3312, which requires 104 drive boards to drive (control 208 PJN). Therefore, a new generation of embedded electronic jacquard system with simple structure and high integration is the development direction of warp knitting machine control system. In order to overcome low reliability and maintenance difficulty of the traditional warp knitting machine electronic jacquard control system with complex multicard structure, our previous work in [23] integrates the microcontroller unit (MCU), driver circuit, DC powers, and communication interfaces with the PJN. Such structure not only greatly reduces the jacquard control system's size but also improves the reliability and the ability of resisting noise interference and vibration.

This article focuses on a design method of an adaptive boost energy-saving fuzzy control system driven by a finite state machine for the PJN. First of all, the electromechanical dynamics model of the PJN was established, and the mathematical relationship between the positive and negative offset displacement of the PJN and the applied voltage was analyzed. Secondly, a fuzzy PI double closed-loop control algorithm driven by a finite state machine is proposed. Third, we construct the driver circuit of the PJN based on MOSFET, which integrates an adaptive self-boost circuit of the highvoltage power supply with the working circuit of the PJN.

\section{Piezoelectric Ceramic Dynamic Model}

The PJN generally consists of two Pb-based Lanthanumdoped Zirconate Titanate (PZT) piezoelectric ceramic wafers and a glass fiber ceramic substrate, as shown in Figure 1.

$l$ is the length of the PJN, $c=2 a+b$ is the thickness of the PJN, $a$ is the thickness of PZT piezoelectric ceramic wafers, $b$ is the thickness of the glass fiber ceramic substrate, $\gamma=$ $a+0.5 b$ is half the thickness of the PJN, $r$ is the radius of curvature, and $\theta$ is the chord center angle. According to the inverse piezoelectric effect, the PJN uses the applied voltage $U_{\text {PJN }}$ to produce a vertical deflection deformation (upper PZT contraction, lower PZT extension) in the vertical direction proportional to the electric field strength and produces a positive and negative offset to achieve the jacquard effect. The bending and stretching amount $\Delta l$ of the PJN is

$$
\Delta l=d_{31} U_{\mathrm{PJN}} \frac{l}{\gamma},
$$

where $d_{31}$ is the piezoelectric strain constant.

Next, we consider the mathematical relationship between the bending and expansion of the PJN and its applied voltage $U_{\mathrm{PJN}}$. It can be seen from Figure 1 that

$$
\left\{\begin{array}{l}
r \theta=l+\Delta l, \\
(r-2 \gamma) \theta=l-\Delta l .
\end{array}\right.
$$

From equation (2), we can get $r=(l+\Delta l) \gamma / \Delta l$ and $\theta=\Delta l / \gamma$. Considering $l+\Delta l \approx l$, it can be concluded that the displacement of the positive and negative offset of the PJN is

$$
\begin{aligned}
\Delta \lambda & \approx r(1-\cos \theta), \\
& =\frac{(l+\Delta l) \gamma}{\Delta l}\left[1-\cos \frac{\Delta l}{\gamma}\right], \\
& =\frac{\gamma^{2}}{d_{31} U_{\mathrm{PJN}}}\left[1-\cos \left(\frac{d_{31} l U_{\mathrm{PJN}}}{\gamma^{2}}\right)\right], \\
& \approx \frac{1}{2} d_{31} U_{\mathrm{PJN}}\left(\frac{l}{\gamma}\right)^{2} .
\end{aligned}
$$

From equation (3), it can be seen that the displacement of the PJN positive and negative offset is proportional to the applied voltage $U_{\mathrm{PJN}}$, proportional to the square of its length $l$, and inversely proportional to the square of the thickness $c$.

\section{PJN Drive Circuit Model}

The drive circuit model includes six MOSFETs such as $V_{1}, V_{2}$, and $V_{31} \sim V_{i 1}(i=3,4,5,6)$. There are 9 diodes, which are $\mathrm{D}_{1} \sim D_{5}, \mathrm{D}_{31} \sim D_{i 1}$, and $D_{31}{ }^{\prime} \sim D_{i 1}{ }^{\prime}, i=3,4,5,6$. There is an energy storage inductor $L$ and a PJN. In the driving circuit, $V_{31} \sim V_{i 1}$ and PJN constitute a double-arm bridge circuit, as shown in Figure 2. The low-voltage power supply voltage is $U_{\mathrm{d}}$ (usually $24 \mathrm{~V}$ power supply), and the high-voltage power 


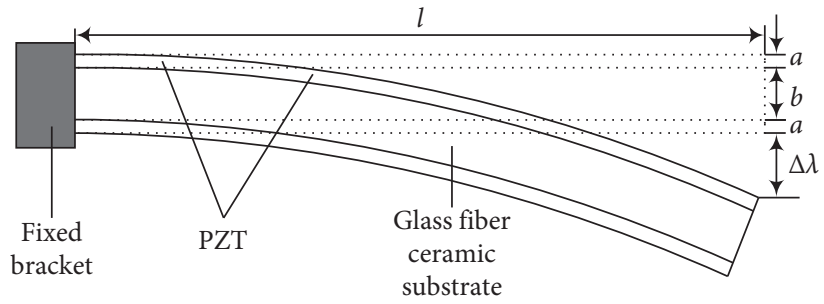

Figure 1: Model of the PJN

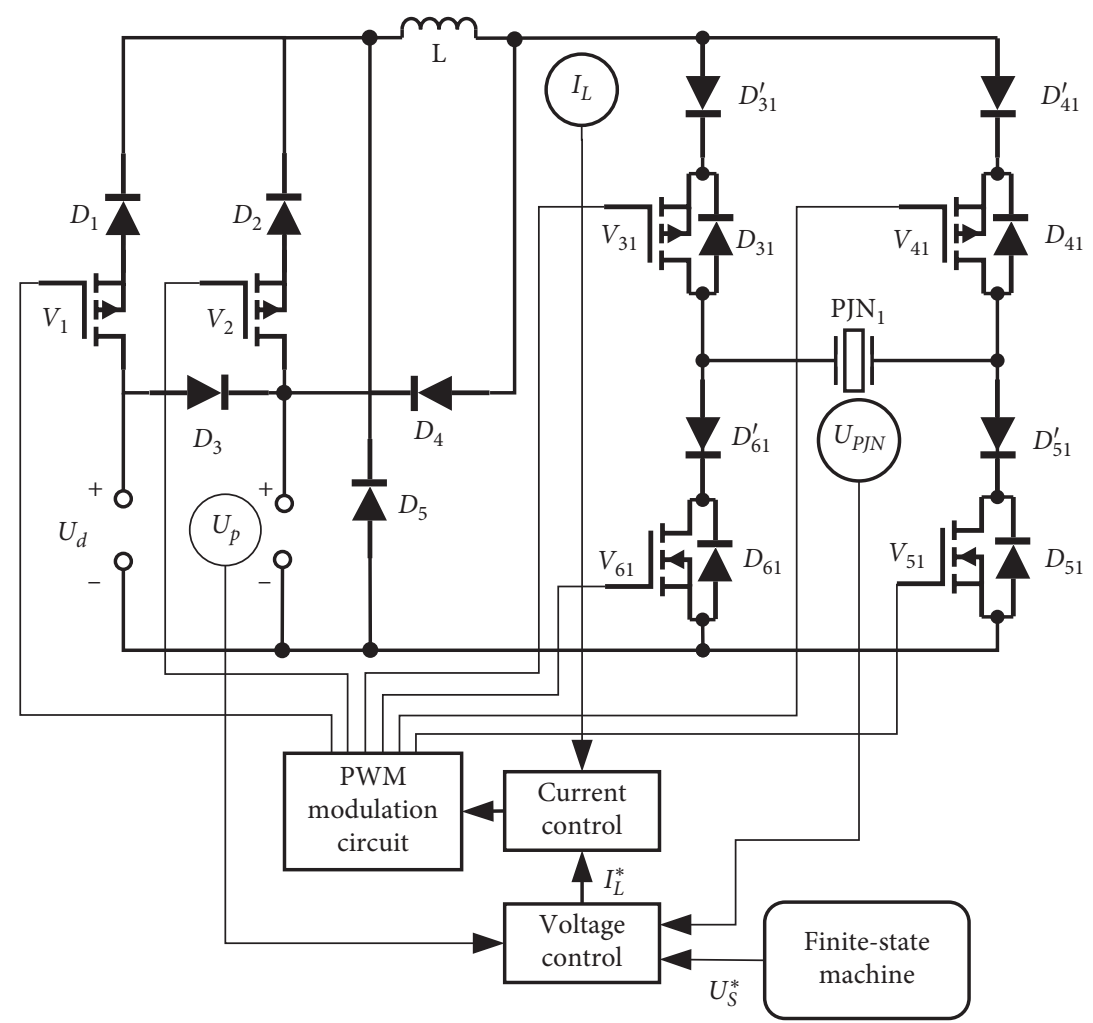

Figure 2: Drive circuit model of the PJN.

supply voltage is $U_{\mathrm{p}}$ (usually DC $100 \mathrm{~V} \sim 200 \mathrm{~V}$ ). The turn-on and turn-off of the MOSFETs are driven by a high-frequency PWM signal. The PWM signal is obtained by modulating the triangular carrier by the control signal output by the voltage and current double closed-loop controller. The logic switching of the initial boosting, high-frequency boosting, and high-voltage driving modes of the drive circuit is completed by the finite-state machine.

\section{PJN Driving Circuit Working Mode Analysis}

The complete working process of the drive circuit of the PJN includes 6 working modes, that is, $S_{0} \sim S_{5}$, as shown in Figure 3. The key waveform of the drive circuit is shown in Figure 4 . The switching condition $c_{i j}$ between modes is determined by the combination of the production process of the warp knitting machine and the characteristics of the driving circuit. The equivalent circuit in different modes is shown in Figure 5.

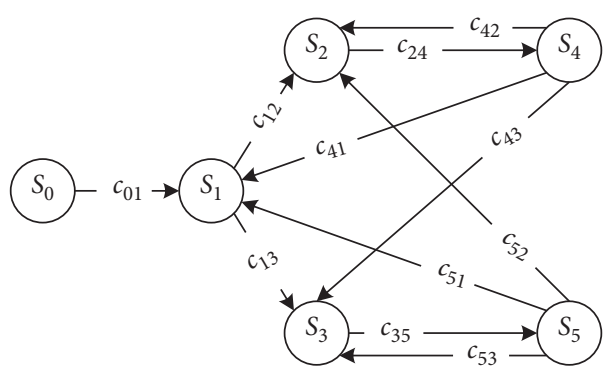

Figure 3: The state transition process of 6 modes.

4.1. The Initial Boost Mode $S_{0}$ of the High-Voltage Power Supply. All MOSETs are turned off through the input control terminal, and the low-voltage power supply $U_{d}$ charges the high-voltage power supply $U_{p}$ through the diode $\mathrm{D}_{3}$ until $U_{p} \geq U_{d}-U_{D D}$. $U_{\mathrm{DD}}$ is the forward voltage drop of the diode $\mathrm{D}_{3}$. The mode $S_{0}$ is shown in Figure 5(a). When 


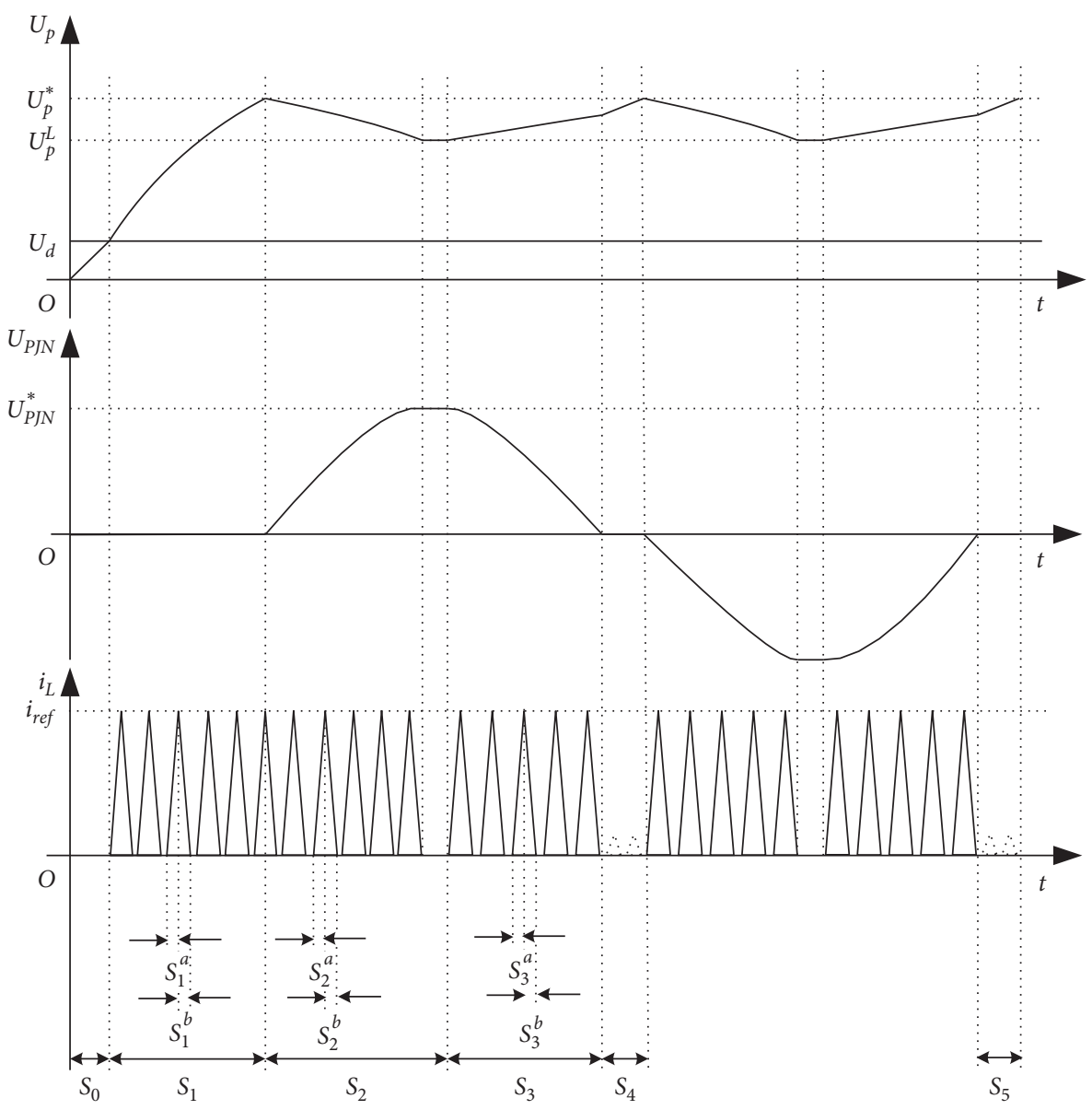

FIgURE 4: The key waveform of the drive circuit of the PJN.

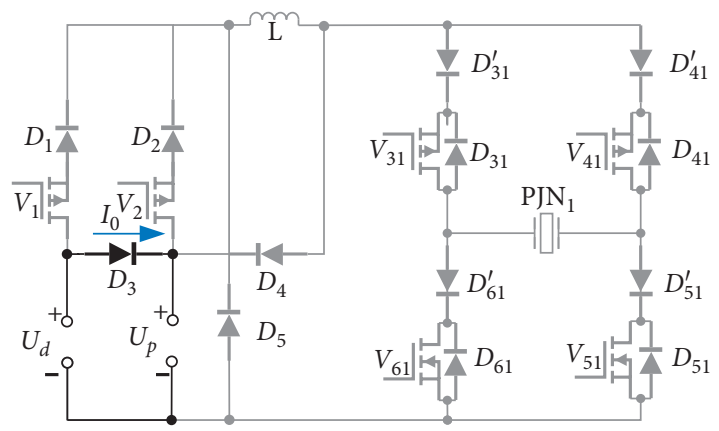

(a)

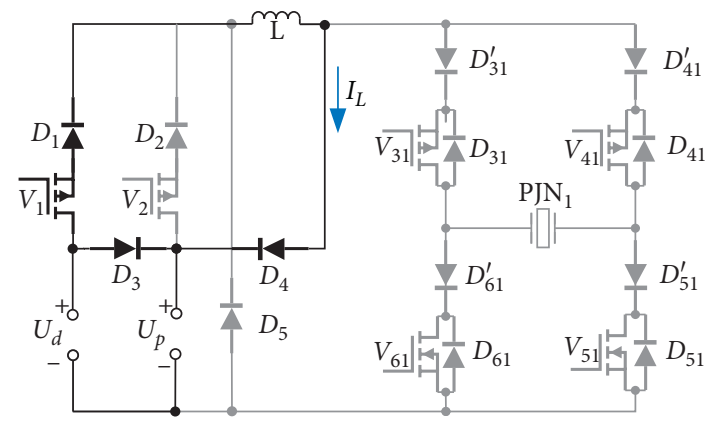

(c)

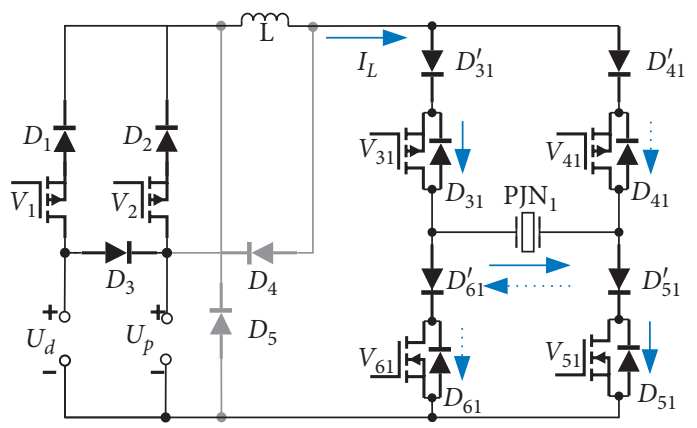

(b)

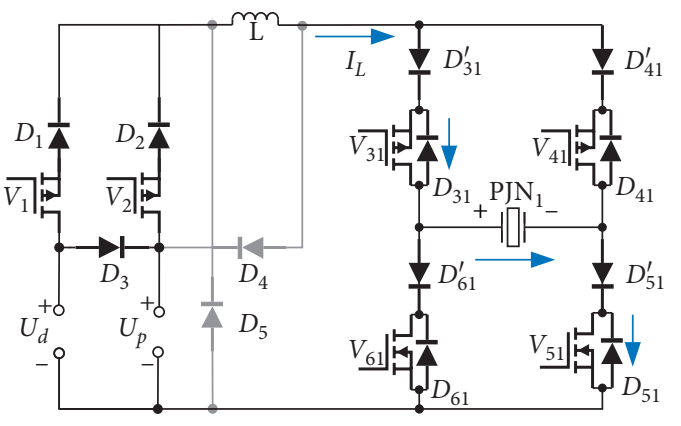

(d)

Figure 5: Continued. 


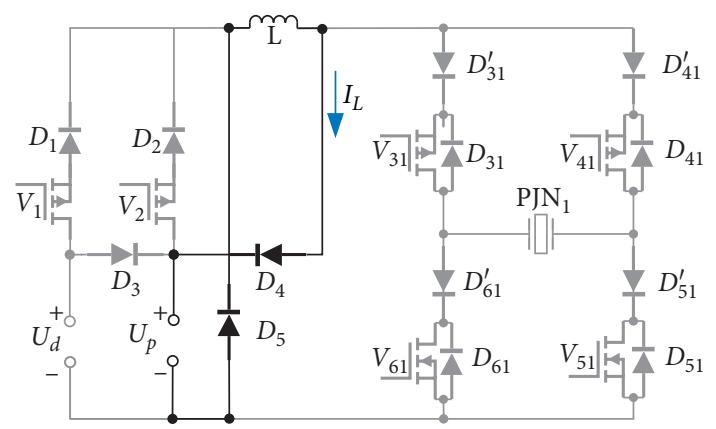

(e)

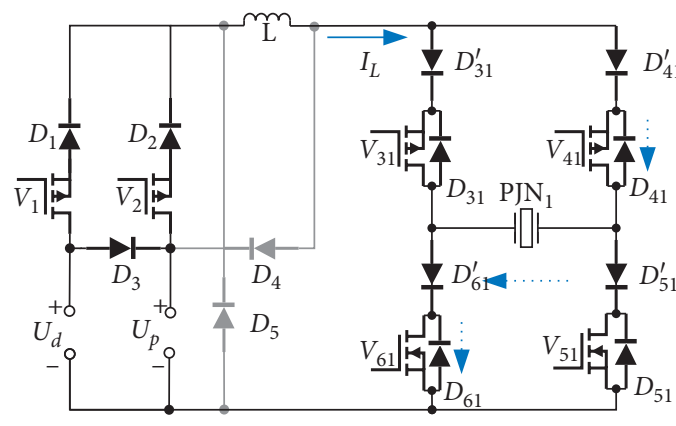

(f)

Figure 5: Equivalent circuits in different modes. (a) Initial boost. (b) The inductor (L) charge. (c) Inductor L discharge and energy recovery. (d) $U_{d}$ and $U_{p}$ charge forward to the PJN. (e) Inductor L discharge. (f) $U_{d}$ and $U_{p}$ charge reversely to the PJN.

$U_{p} \geq U_{d}-U_{D D}$, the condition $c_{01}=1$ satisfied, and the drive circuit enters the mode $S_{1}$ from the mode $S_{0}$.

\subsection{High-Frequency Boost Mode $S_{1}$ of High-Voltage Power} Supply. $U_{p}$ is charged through $U_{d}$ and $L$ until $U_{p}$ reaches the rated voltage $U_{p}^{*}$. Real-time detection of $U_{p}$ is carried out during the boost process; if $U_{p}>=U_{p}^{*}$ is satisfied, the charging boost process ends. This process is realized by highfrequency cyclic switching of the two submodes $S_{1}^{a}$ and $S_{1}^{b}$, as shown in Figures 3(b) and 3(c). In mode $S_{1}^{a}, U_{d}$ charges and stores energy for L. During the charging process of $L$, the two arms of the double-arm bridge composed of $V_{31} V_{41} V_{51}$, and $V_{61}$ work alternately at high frequencies, so as to ensure that the charging and discharging energy of the PJN are complementary and remain in a balanced position. In the mode $S_{1}^{a}, i_{L}$ rises according to the following formula:

$$
i_{L}=\frac{U_{d}}{L} t+i_{d}(0)
$$

When $i_{L}>i_{L}^{*}\left(i_{L}^{*}\right.$ is the reference set current), the circuit is switched from $S_{1}^{a}$ to $S_{1}^{b}$, the inductor $L$ is discharged to ensure $i_{\mathrm{L}} \leq i_{L}^{*}$, and the energy in the $L$ is recovered to $U_{\mathrm{p}}$. In mode $S_{1}^{b}$, $i_{L}$ drops according to the following formula:

$$
i_{L}=-\frac{U_{p}-U_{d}}{L} t+i_{L}^{*}
$$

According to the conservation of energy, we can get

$$
U_{d} I_{L} T_{a}=\left(U_{p}-U_{d}\right) I_{L} T_{b}
$$

where $I_{L}$ is the average value of $i_{L}, T_{a}$ is the total time occupied by submode $S_{1}^{a}$ intermittently in a mode $S_{1}$, and $T_{b}$ is the total time occupied by submode $S_{1}^{b}$ intermittently in a mode $S_{1}$.

According to formula (6), we can get

$$
U_{p}=\frac{T_{a}+T_{b}}{T_{b}} U_{d}
$$

When $U_{p}>=U_{p}^{*}$, the condition $c_{12}=1$ or $c_{13}=1$ satisfied, and the drive circuit enters $S_{2}$ (positive offset) or $S_{3}$ (negative offset) from $S_{1}$.
4.3. Positive Offset Mode $S_{2}$ and Positive Offset Mode $S_{3}$. Mode $S_{2}$ : assuming that the rated driving voltage of the PJN that meets the process requirements is $U_{\mathrm{PJN}}^{*}$, the power supply $U_{p}$ positively charges the PJN (reverse piezoelectric effect) until $U_{\mathrm{PJN}} \geq U_{\mathrm{PJN}}^{*}$. This process is realized by highfrequency cyclic switching between the two submodes $S_{2}^{a}$ and $S_{2}^{b}$, as shown in Figures $3(\mathrm{~d})$ and $3(\mathrm{e})$. $U_{\mathrm{PJN}}^{*}$ should meet

$$
U_{\mathrm{PJN}}^{*}=U_{p}^{*} \frac{C_{\mathrm{PJN}}}{C_{p}+C_{\mathrm{PJN}}},
$$

where $C_{\mathrm{PJN}}$ is the equivalent capacitance of the $\mathrm{PJN}$ and $C_{p}$ is the equivalent capacitance of $U_{p}$. It can be seen from equation (8) that, in order to obtain $U_{\mathrm{PJN}}^{*}$ that satisfies the warp knitting process, $U_{p}^{*}>U_{\mathrm{PJN}}^{*}$ must be made in the mode $S_{1}$.

In mode $S_{2}^{a}, i_{L}$ rises according to the following formula:

$$
i_{L}=\frac{U_{p}-U_{\mathrm{PZT}}}{L} t+i_{L}^{*}
$$

In order to ensure $i_{L} \leq i^{*}, L$ discharges in mode $S_{2}^{b}$, and $i_{L}$ decreases according to the following formula:

$$
i_{L}=-\frac{U_{d}}{L} t+i_{L}^{*}
$$

Mode $S_{3}$ : assuming that the rated driving voltage of the PJN that meets the process requirements is $-U_{\mathrm{PJN}}^{*}, U_{p}$ will reversely charge the PJN until $U_{\mathrm{PJN}} \leq-U_{\mathrm{PJN}}^{*}$, and the two submodes $S_{3}^{a}$ and $S_{3}^{b}$ (as shown in Figures 3(f) and 3(e)) work similar to $S_{2}^{b}$ and $S_{2}^{b}$.

4.4. Equilibrium Mode $S_{4}$ and $S_{5}$. According to the warp knitting process, the PJN will return to the equilibrium modes $S_{2}$ or $S_{3}$ after meeting the conversion conditions $c_{42}=$ 1 or $c_{42}=1$ after working for a time interval in $S_{4}$ or $S_{5}$. The next action of the PJN in the equilibrium mode will depend on the warp knitting process and the state of $U_{p}$. For example, if the PJN is currently in the balanced mode $S_{4}$ and $U_{p}>=U_{p}^{*}$, the next step of the warp knitting process will continue to shift positively, and the transition condition $c_{42}=1$ of the finite state machine is triggered, causing the drive circuit to transfer to the mode $S_{2}$. Note that if $U_{p}<U_{p}^{*}$, 
you need to trigger $c_{41}=1$ to return to mode $S_{1}$ to charge $U_{\mathrm{p}}$ to compensate for the energy lost during driving the PJN. After $U_{p}>=U_{p}^{*}$, the charging and boosting process ends, and $U_{\mathrm{p}}$ continues to drive the PJN according to the warp knitting process.

\section{Fuzzy PI Control Algorithm Driven by the Finite State Machine}

Generally, the circuit for multimode switching operation needs to adopt an independent controller in each working mode. In this paper, a finite-state machine is used to drive a fuzzy PI controller so that it can adapt to different circuit modes, as shown in Figure 6.

The fuzzy PI controller adopts double closed-loop control, where $U_{S}^{*}=\left\{U_{p}^{*}, U_{\mathrm{PJN}}^{*},-U_{\mathrm{PJN}}^{*}\right\}$. The inner loop of the fuzzy PI controller is the antisaturation current PI control loop, which outputs the signal $U_{r}^{\prime} \in\left[-U_{r}^{\prime}, U_{r}^{\prime}\right]$ through the conversion factor $\alpha$, and generates the PWM signal $K$ to drive the MOSFETs by modulating the triangular wave. The outer loop is an antisaturation voltage fuzzy PI control loop, and the output is $U_{r} \in\left[-U_{r}, U_{r}\right]$. When the deviation $e_{u}$ is large, the outer loop output $U_{r}$ is always saturated, the voltage loop is equivalent to an open loop, and the system becomes a current regulation system based on the conversion voltage $U_{i}^{*}$ of $i_{L}^{*}$, basically keeping the $I_{L}$ constant. When the deviation $e_{u}$ is close to zero, it indicates that the target voltage is basically reached, $U_{r}$ gradually exits the saturation state, and $U_{i}^{*}$ and $i_{L}$ drop rapidly.

The proportional and integral adjustment parameters of the outer-loop fuzzy PI controller are $k_{p}=k_{p 0}+\Delta k_{p}$ and $k_{i}=k_{i 0}+\Delta k_{i}$, respectively, where $k_{p 0}$ and $k_{i 0}$ are the initial values, and $\Delta k_{p}$ and $\Delta k_{i}$ are the fuzzy control variables. The establishment of fuzzy control rules requires full consideration of the characteristics of the PJN drive circuit. For example: when the mode of the circuit is $S_{1}\left(U_{S}^{*}=U_{p}^{*}, e_{u}=U_{p}^{*}-U_{p}\right)$ and the voltage difference $e_{u}$ and $\Delta e_{u}$ is large, it shows that the deviation of $U_{p}$ and $U_{\mathrm{p}}^{*}$ is large, so $\Delta k_{p}$ and $\Delta k_{i}$ should be increased to achieve the purpose of quickly reducing the $e_{u}$. The fuzzy rule table is shown in Table 1 , which contains 18 fuzzy rules in the form of "if $A_{\mathrm{i}}$ and $B_{\mathrm{i}}$, then $C_{i}^{\prime}$. In order to reduce the computational complexity of the proposed fuzzy PI controller, we only use 18 fuzzy control rules. In future work, we will use the offline generated fuzzy control table to avoid online real-time fuzzy inference which will further improve the operating efficiency of the control system.

In this paper, the fuzzy domain of input and output is divided into three fuzzy subsets $\{N, Z, P\}$. When the deviation is small, the triangular membership function is used to improve the control sensitivity. The analytical formula is shown in equation (11), where $a$ is the midpoint of the fuzzy subset and $c$ is the distance from the midpoint to the two ends of the fuzzy subset. When the deviation is large, the $\mathrm{s}$-shaped membership function is used, and the analytical formula is as in equation (12).

$$
\begin{aligned}
& u_{t}(x)= \begin{cases}\left.\frac{0}{c}, a+c\right) & x \leq(a-c), \\
u_{s}(x) & (a-c) \leq x \leq a, \\
\frac{(x+a+c)}{c}, & a \leq x \leq(a+c), \\
0, & (a+c) \leq x, \\
2\left[\frac{(x-a+c)}{2 c}\right]^{2}, & (a-c) \leq x \leq a, \\
1-2\left[\frac{(x-a-c)}{2 c}\right]^{2}, & a \leq x \leq(a+c), \\
1, & (a-c),\end{cases} \\
& \hline \begin{array}{ll}
0, & (a+c) \leq x .
\end{array}
\end{aligned}
$$

The relationship between the clarification variables $e_{u}$ and $\Delta e_{u}$ and the fuzzy variables $E_{u}$ and $\Delta E_{u}$ is

$$
\begin{gathered}
E_{u}=e_{u} \cdot k_{e}, \\
\Delta E_{u}=\Delta e_{u} \cdot k_{\Delta e},
\end{gathered}
$$

where $k_{e}$ and $k_{\Delta e}$ are the quantization factors of $E_{u}$ and $\Delta E_{u}$, respectively.

The input and output membership functions of the fuzzy inference are shown in Figures 7(a), 8(b), and 8(c). The fuzzy adaptive adjustment of $k_{p}$ and $k_{i}$ is shown in Figure $8(\mathrm{~d})$. The relationship between PI parameter correction variables $\Delta k_{p}$ and $\Delta k_{i}$ and $e_{u}$ and $\Delta e_{u}$, respectively, is shown in Figure 9.

The Mamdani reasoning rule is used to perform fuzzy reasoning as follows:

$$
\left\{\begin{array}{l}
R=U_{i=1}^{n} R_{i}=U_{i=1}^{n}\left(A_{i} \times B_{i}\right) \times C_{i}, \\
\mu_{R i}=\min \left\{\mu_{A i}\left(E_{u}\right), \mu_{B i}\left(\Delta E_{u}\right), \mu_{C i}(U)\right\}, \\
\mu_{C}(U)=\max \left\{\min \left\{\mu_{A \times B}\left(E_{u}, \Delta E_{u}\right), \mu_{R}\left(E_{u}, \Delta E_{u}, U\right)\right\}\right\} .
\end{array}\right.
$$

We use the center of gravity method with smoother output, simpler calculation, and higher accuracy to perform the following defuzzification:

$$
\left\{\begin{array}{l}
z^{*}=\frac{\int_{x_{1}}^{x_{2}} z \cdot \mu_{C}(z) \mathrm{d} z}{\int_{x_{1}}^{x_{2}} \mu_{C}(z) \mathrm{d} z} \\
U=\left[x_{1}, x_{1}\right] \\
z^{*}=k_{p}, k_{i} .
\end{array}\right.
$$




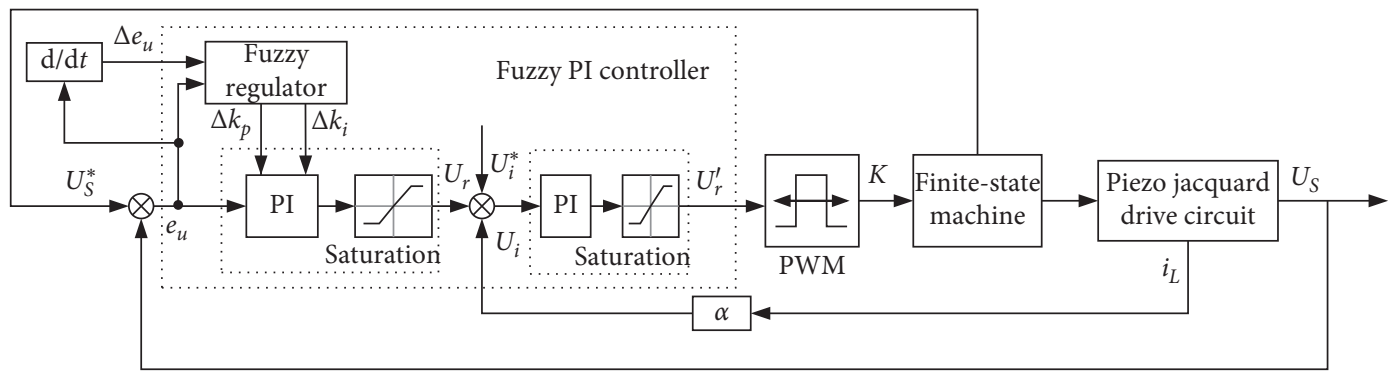

Figure 6: The fuzzy PI controller driven by the finite state machine.

TABLE 1: Fuzzy control rules of $\Delta k_{p}, \Delta k_{i}$.

\begin{tabular}{lccc}
\hline$\Delta e_{u}$ & & $e_{u}$ & \\
& $\mathrm{~N}$ & $\mathrm{Z}$ & $\mathrm{P}$ \\
\hline $\mathrm{N}$ & $\mathrm{N} / \mathrm{Z}$ & $\mathrm{N} / \mathrm{Z}$ \\
$\mathrm{Z}$ & $\mathrm{N} / \mathrm{P}$ & $\mathrm{P} / \mathrm{P}$ & $\mathrm{P} / \mathrm{P}$ \\
$\mathrm{P}$ & $\mathrm{P} / \mathrm{Z}$ & $\mathrm{P} / \mathrm{Z}$ & $\mathrm{P} / \mathrm{Z}$ \\
\hline
\end{tabular}

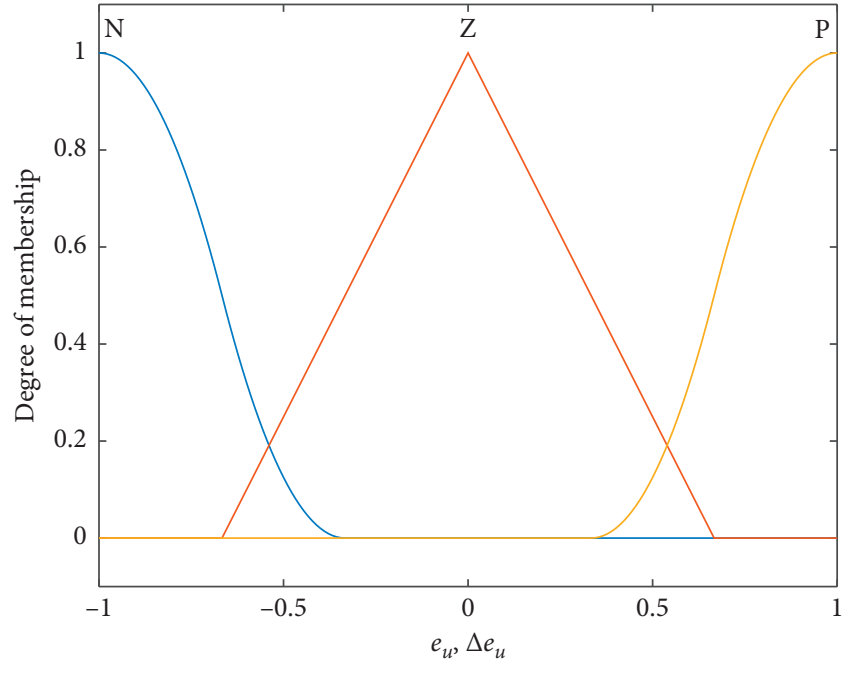

(a)

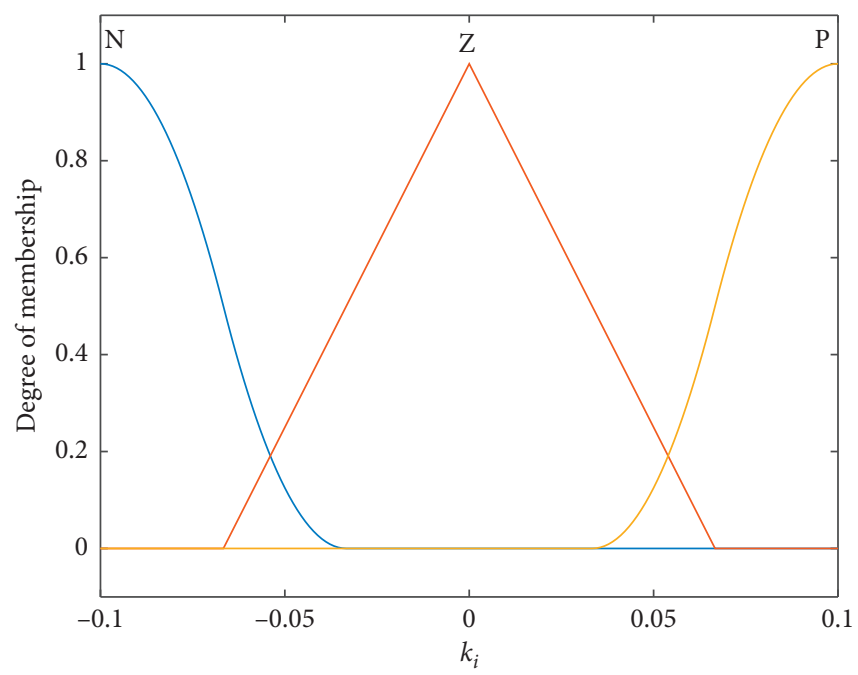

(c)

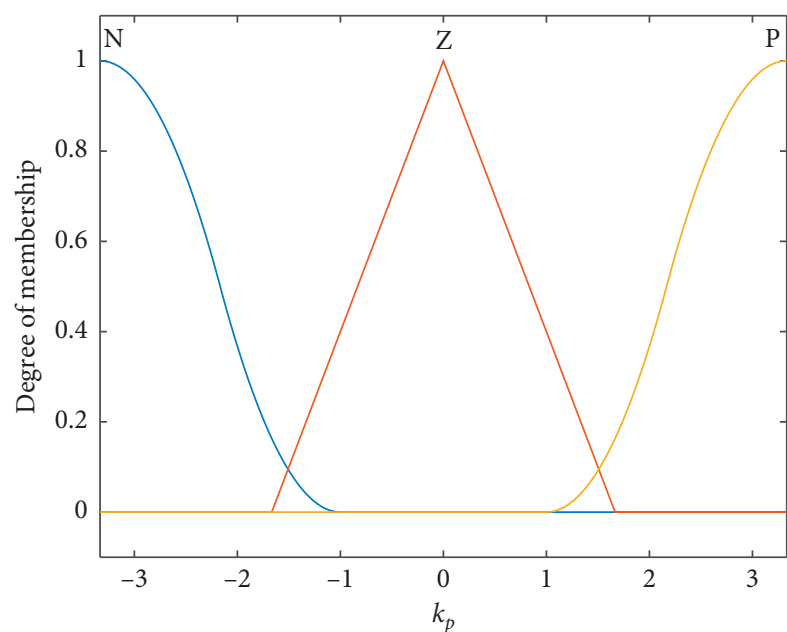

(b)
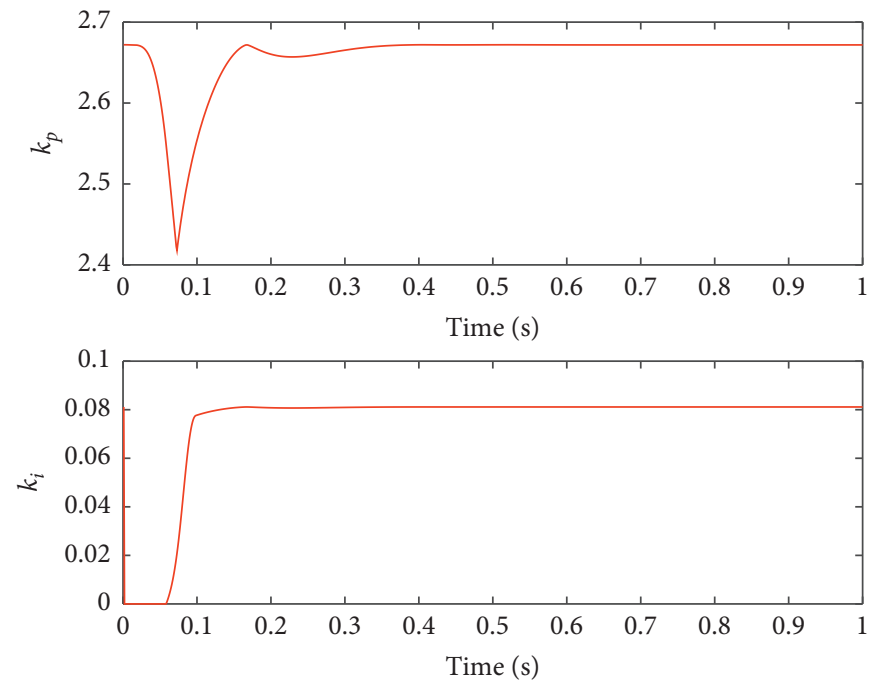

(d)

Figure 7: Membership function of fuzzy controller input and output and the adaptive adjustment of $k_{p}$, $k_{i}$. (a) Membership function of $e_{u}, \Delta e_{u}$. (b) Membership function of $k_{p}$. (c) Membership function of $k_{i}$. (d) Adaptive adjustment of $k_{p}, k_{i}$. 


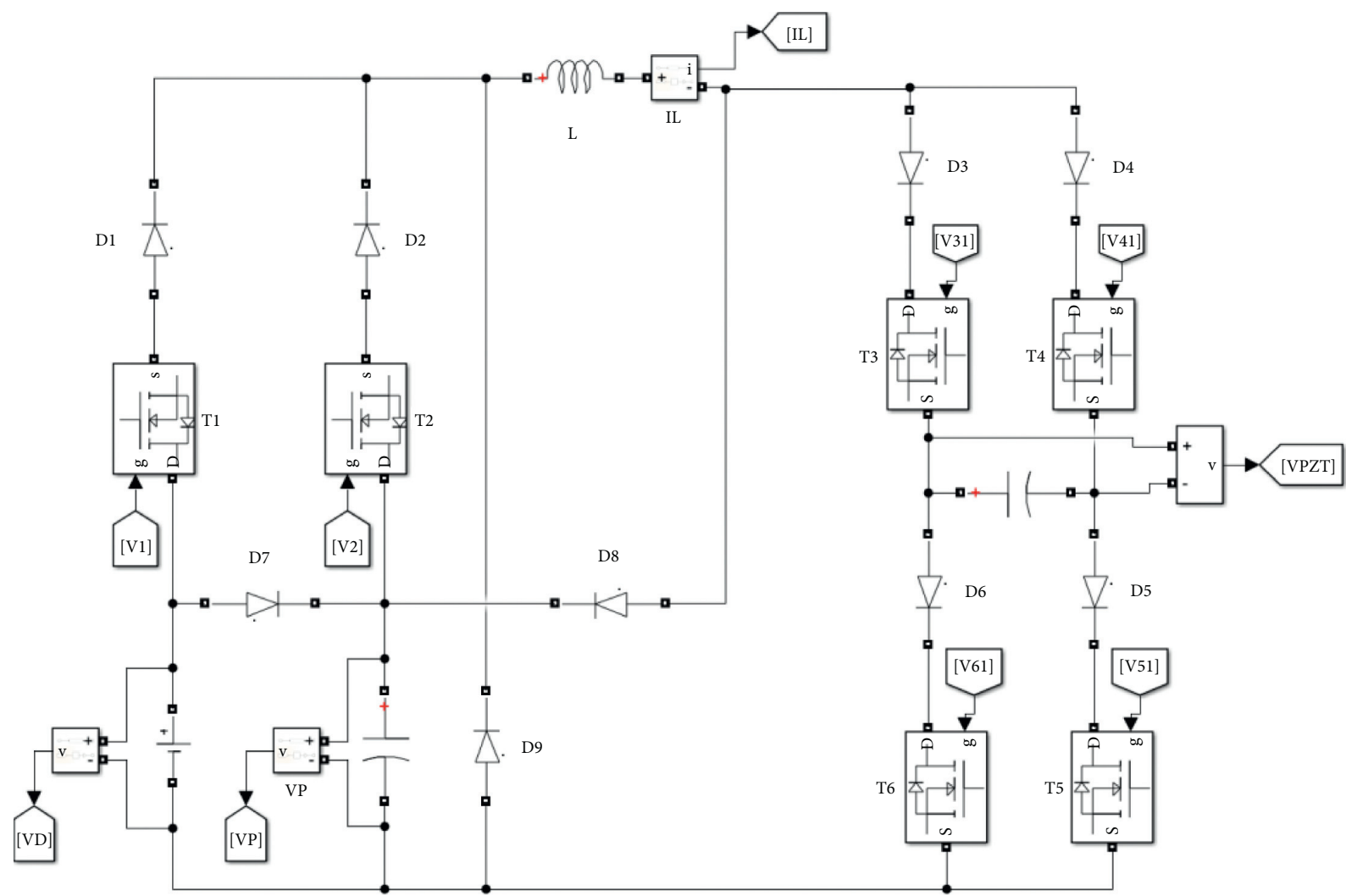

FIGURE 8: Driving circuit of the PJN.

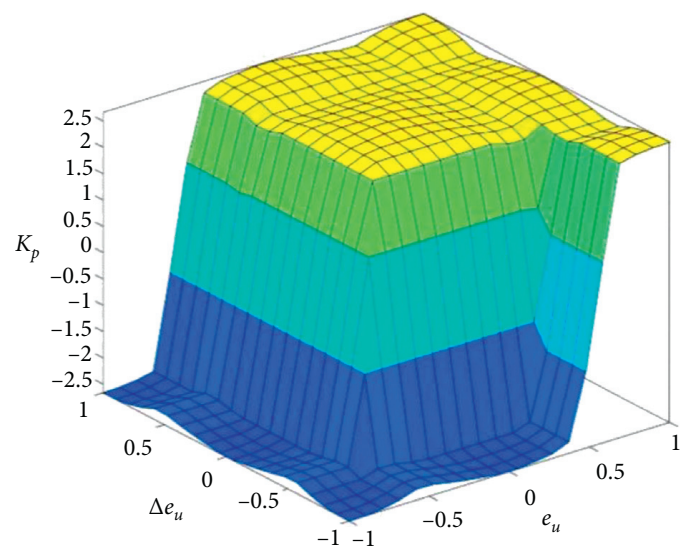

(a)

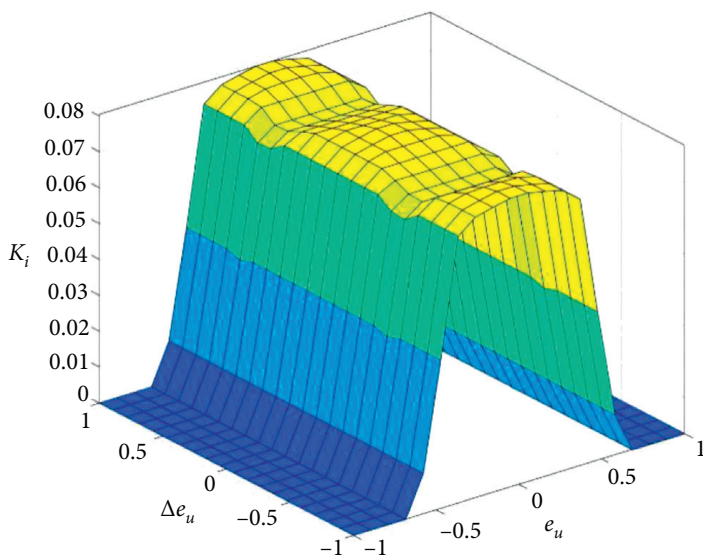

(b)

Figure 9: Diagram of the relationship between inputs and outputs in fuzzy reasoning. (a) Correspondence between $e_{u}$, $\Delta e_{u}$ and $k_{p}$. (b) Correspondence between $e_{u}, \Delta e_{u}$ and $k_{i}$.

\section{Simulation}

In order to verify the effectiveness of the design strategy of the adaptive boost energy-saving fuzzy control system driven by the finite-state machine, a PJN drive circuit was built in MATLAB/Simulink, as shown in Figure 8. $C_{p}=1 \mu \mathrm{F}, C_{\mathrm{PJN}}=30 \mathrm{nF}, L=30 \mathrm{mH}, V_{d}=24 \mathrm{~V}, U_{p}^{*}=205 \mathrm{~V}$, $U_{\mathrm{PJN}}^{*}=150 \mathrm{~V}$, and $i_{L}^{*}=20 \mathrm{~mA}$.
In order to ensure that $U_{p}$ and $U_{\mathrm{PJN}}$ can follow the given values $U_{p}^{*}$ and $U_{\text {PJN }}^{*}$ smoothly and quickly, a double closedloop control system is established, as shown in Figure 10. Each module is encapsulated in a subsystem. It can be seen from Figure 10 that the drive circuit of the PJN is driven by a finite-state machine, and the PWM signal in the finite-state machine needs to be adjusted automatically by the fuzzy PI controller. 


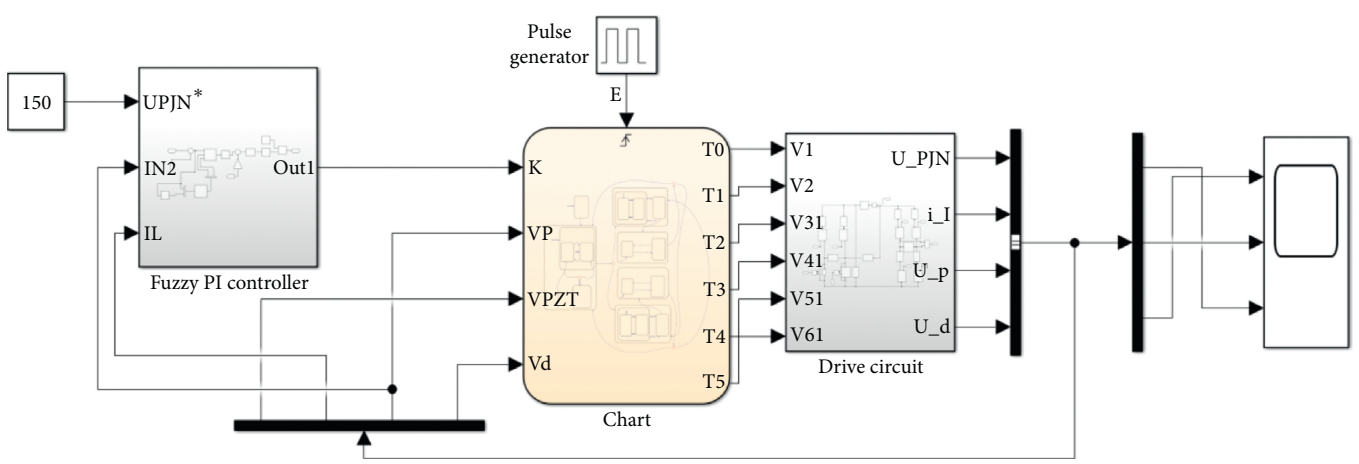

FIgUre 10: Adaptive boost energy-saving fuzzy control system of the PJN.

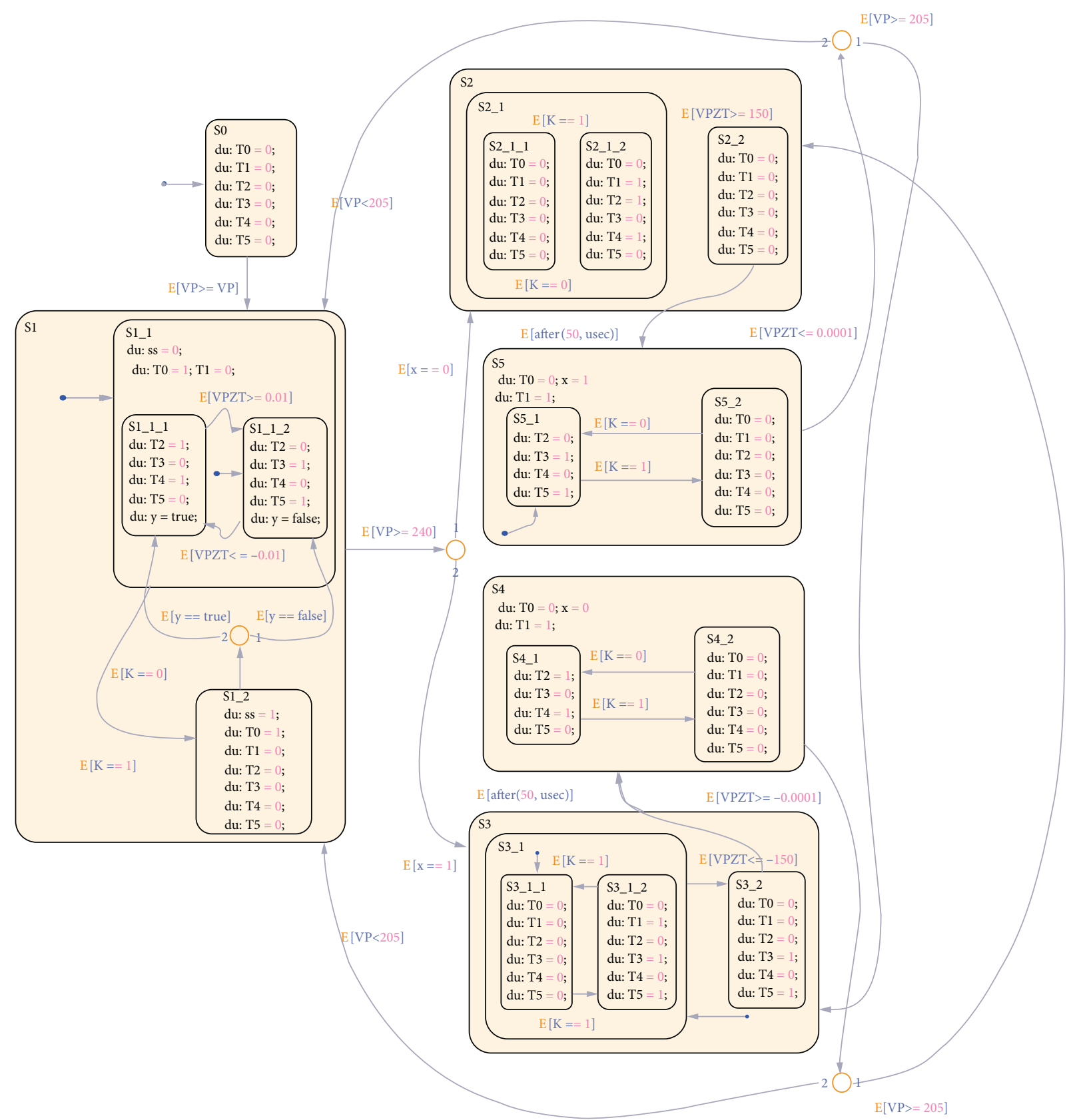

Figure 11: Finite-state machine model. 

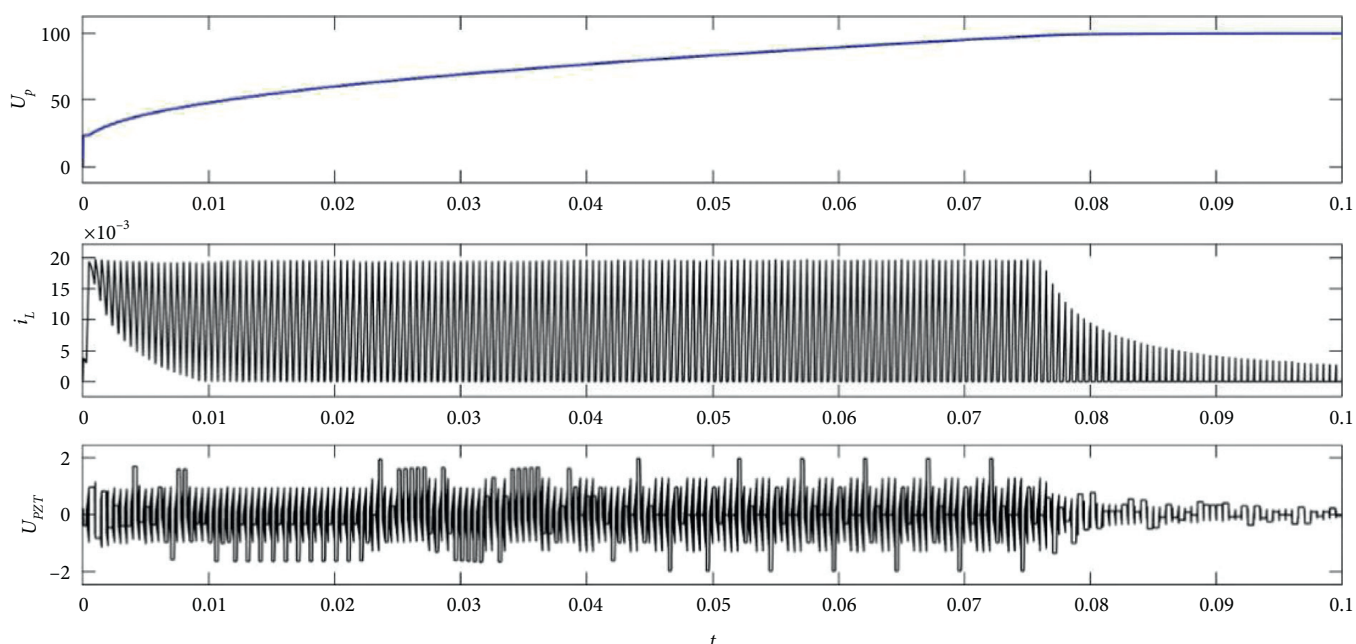

(a)
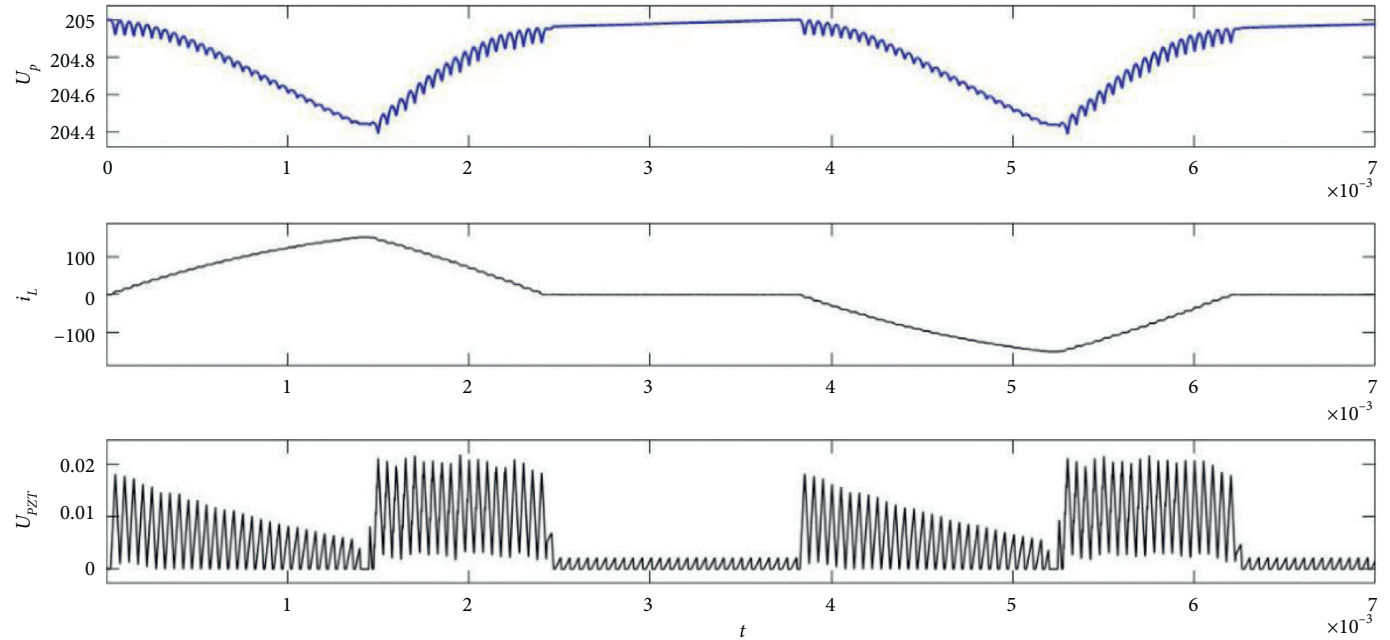

(b)

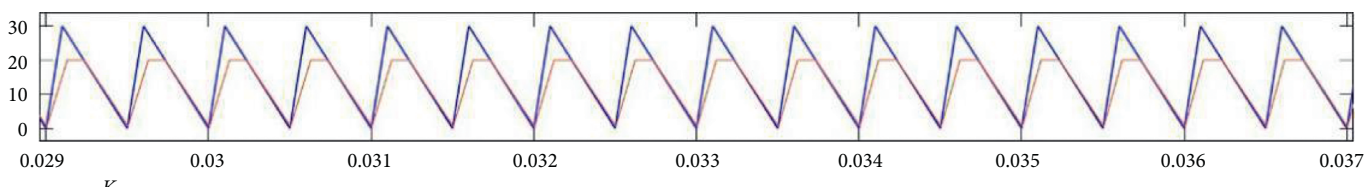

Triangle wave

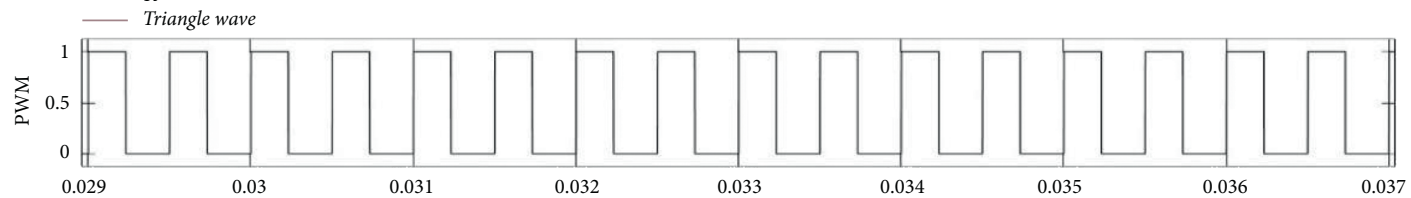

(c)

Figure 12: Curve of $U_{\mathrm{PJN}}, U_{P}, i_{L}$, and PWM. (a) Curve of $U_{\mathrm{PJN}}, U_{P}$, and $i_{L}$ in the process of adaptive boost. (b) Curve of $U_{\mathrm{PJN}}, U_{P}$, and $i_{L}$ in the process of jacquard work. (c) PWM working waveform.

The finite-state machine model is shown in Figure 11. It can be seen from Figure 11 that the output voltage of the fuzzy PI controller generates the PWM signal of the MOSFETs in the driving circuit by modulating the triangular wave, which determines the frequency of the current $i_{L}$ in the circuit. The finite-state machine realizes the logic switching between the various modes of the circuit.
The adaptive self-boost performance, jacquard needle drive function, and energy-saving effect of the PJN drive circuit are shown in Figures 12(a) and 12(b), and the PWM waveform generation process is shown in Figure 12(c). It can be seen from Figure 12(a) that it only took $100 \mathrm{~ms}$ for $U_{P}$ to boost from $0 \mathrm{~V}$ to $100 \mathrm{~V}$. During $U_{P} \ll 100 \mathrm{~V}$, the voltage outer loop is in a saturated state, and only the current inner 
loop control circuit, by setting $U_{r}^{\prime} \in\left[0_{r}, 20\right]$, guarantees $i_{L} \leq 20 \mathrm{~ms} . U_{r} \in[-2,2]$ is set in the simulation process, and during $U_{P} \geq 98 \mathrm{~V}$, the voltage outer loop starts to work and smoothly and quickly adjust $U_{P}$ to boost to $100 \mathrm{~V}$. Figure 12(a) also shows that the decay speed of $i_{L}$ in the substate $S_{1}^{b}$ of the boost mode $S_{1}$ continues to increase. The average voltage $\bar{U}_{\mathrm{PJN}}$ of the $U_{\mathrm{PJN}}$ is zero, which ensures that the PJN is stationary at the equilibrium position. Figure 12(b) shows the positive and negative offset of Set PJN for one cycle under the conditions of $U_{p}^{*}=205 \mathrm{~V}$ and $U_{\mathrm{PJN}}^{*}=150 \mathrm{~V}$. From Figure 12(b), it can be seen that the positive offset time of PJN is $T_{+}=1.5 \mathrm{~ms}$, the negative offset time is $T_{-}=1 \mathrm{~ms}$, the time to stay in the equilibrium position is $T_{O}=1.5 \mathrm{~ms}$, and the total time of an offset cycle is $T_{T}=T_{L}+T_{R}+T_{O}=4 \mathrm{~ms}$, which satisfies the process requirements $3 \sim 20 \mathrm{~ms}$, which shows that the proposed driving strategy is feasible. It can be seen from Figure 12(b) that, in the $T_{-}$phase $\left(S_{1}^{b}\right.$ mode), the circuit recovers the energy stored in $L$ through the piezoelectric effect of the PJN, which has energy-saving performance. Although energy is recovered in phase $T_{-}$, the high-voltage power supply $U_{P}$ still has a small loss $\left(U_{P}<205 \mathrm{~V}\right)$, so in phase $S_{1}\left(\operatorname{mode} S_{4}\right)$, it returns to the boost mode $S_{1}$, trying to boost up to $U_{P}$.

\section{Conclusions}

This paper integrates the self-boosting circuit of PJN highvoltage power supply with the PJN high-frequency working circuit to improve the circuit integration. The logic switching sequence of each functional mode of the circuit is dispatched by a finite-state machine. The adopted PI fuzzy double closed-loop control algorithm can realize the controlled voltage value of the circuit in each mode smoothly and quickly reaching the target value. The new PJN drive circuit designed in this paper uses energy storage inductors instead of the current limiting resistors of the traditional drive circuit, making the circuit have a self-boosting function, without external high-voltage power supply, only lowvoltage power supply, effectively reducing the complexity of the circuit. The energy storage capacity of the inductor can be used for energy recovery, achieving energy saving and low power consumption. The design strategy proposed in this paper provides a theoretical basis for the design of the embedded jacquard miniaturized control system of the warp knitting machine. It would be interesting to consider Takagi-Sugeno fuzzy neural networks to analyze the stability and provide self-learning capabilities in the control problem of the driving circuit of the PJN for future work.

\section{Data Availability}

The data used to support the findings of this study are included within the article.

\section{Conflicts of Interest}

The authors declare that there are no conflicts of interest regarding the publication of this paper.

\section{Acknowledgments}

This work was supported by the Key Industrial Guidance Projects of Fujian Province (2019H0034).

\section{References}

[1] A. J. Mohammad, R. Micky, and A. Omar, "Further results on hysteresis compensation of smart micropositioning systems with the inverse Prandtl-ishlinskii compensator," IEEE Transactions on ControlSystem Technology, vol. 24, no. 2, pp. 428-439, 2016.

[2] Y. Wang, S. Wu, L. Xu, and Y. Zeng, "A new precise positioning method for piezoelectric scanner of AFM," Ultramicroscopy, vol. 196, pp. 67-73, 2019.

[3] Y. Y. Wang, Y. Bian, and H. Guo, "Study of hysteresis modeling method for piezoelectric driver," Journal of Transduction Technology, vol. 32, no. 4, pp. 562-567, 2019.

[4] X. H. Gao, Y. G. Liu, and Z. C. Pei, "Optimization and design for magnetic circuit in giant magnetostrictive actuator," Journal of Harbin Institute of Technology, vol. 48, no. 9, pp. 145-150, 2016.

[5] J. Ravenhorst and R. Akkerman, "A yarn interaction model for circular braiding," Composites Part A: Applied Science and Manufacturing, vol. 81, pp. 254-263, 2016.

[6] N. Gunasekaran, R. Saravanakumar, Y. H. Joo, and H. S. Kim, "Finite-time synchronization of sampled-data T-S fuzzy complex dynamical networks subject to average dwell-time approach," Fuzzy Sets and Systems, vol. 374, pp. 40-59, 2019.

[7] N. Gunasekaran, R. Saravanakumar, M. Syed Ali et al., "Exponential sampled-data control for T-S fuzzy systems: application to Chua's circuit," International Journal of Systems Science, vol. 50, no. 16, pp. 2979-2992.

[8] M. S. Ali, N. Gunasekaran, and Q. Zhu, "State estimation of T-S fuzzy delayed neural networks with Markovian jumping parameters using sampled-data control," Fuzzy Sets and Systems, vol. 306, pp. 87-104, 2017.

[9] N. Gunasekaran and Y. H. Joo, "Robust sampled-data fuzzy control for nonlinear systems and its applications: free-weight matrix method," IEEE Transactions on Fuzzy Systems, vol. 27, no. 11, pp. 2130-2139, 2019.

[10] E. Yucel, M. Syed Ali, N. Gunasekaran, and S. Arik, "Sampleddata filtering of Takagi-Sugeno fuzzy neural networks with interval time-varying delays," Fuzzy Sets and Systems, vol. 316, pp. 69-81, 2017.

[11] C. Liu and Y. Guo, "Modeling and positioning of a PZT precision drive system," Sensors, vol. 17, no. 11, p. 2577, 2017.

[12] X. B. Tan and J. S. Baras, "Adaptive identification and control of hysteresis in smart materials," IEEE Transactions on Automatic Control, vol. 50, no. 6, pp. 827-839, 2005.

[13] Y. Z. Guo and Y. C. Zhu, "Modeling and analysis on hysteresis nonlinear characteristics of the piczoclectrics Stack actuators," Piczoclcctrics \& Acoustooptics, vol. 39, no. 4, pp. 520-524, 2017.

[14] A. Michalak, M. Kuchar, and Z. Mikołajczyk, "Simulation tests of the feeding system dynamics on a warp knitting machine with four needle bars," Fibres \& Textiles in Eastern Europe, vol. 23, no. 112, pp. 127-133, 2015.

[15] D. S. Wang and K. Zhang, Design Principle of Piezoelectric Transducer, pp. 110-112, Wuhan University of Technology Press, Wuhan, China, 2016.

[16] J. Q. Xu, L. L. Ge, C. H. Zong et al., "Design of self-powered power supply of sensor for piezoelectric energy harvesting," 
Piezoelectrics \& Acoustooptics, vol. 41, no. 2, pp. 435-438, 2019.

[17] S. C. Lai, W. Ren, Z. W. He et al., Piezoelectric Ceramic Driving Circuit and Driving Method: China, CN201810812515.X, 2019.

[18] R. Kumaravelu, S. Poornima, and N. Kasthuri, "Woven design data transmission using TTL logic for 128 hooks electronic cardless jacquard," Indian Journal of Fibre \&Textile Research, vol. 40, pp. 100-104, 2015.

[19] O. Peter, "Warp knitting machines," International Textile Bulletin, vol. 49, no. 6, pp. 52-53, 2003.

[20] S. C. Chen, L. Liu, and R. F. Li, "Etc al, Full automation reformation of multibar jacquard raschel warp knitting machine," IOP Conference Series: Materials Science and Engineering, vol. 36, no. 4, pp. 599-603, 2014.

[21] L. Cheng and G. M. Jiang, "The jacquard principle and technology design of the RSJ4/2F warp knitting machine," Knitting Industry, vol. 10, pp. 15-18, 2014.

[22] M. Y. Gao and Y. F. Liu, "Design of the automatic jacquard control system based on STM32F407," in Proceedings of the International Conference on Information Science, Electronics and Electrical Engineering, Denpasar, Bali, Indonesia, October 2019.

[23] W. Ren and S. C. Lai, "Embedded electronic jacquard guide bar: a new approach to warp knitting using the machine jacquard control system," Fibres \& Textiles In Eastern Europe, vol. 26, no. 132, pp. 95-101, 2018. 